\title{
Origin of the Solar Wind and Open Coronal Magnetic Structures
}

\author{
Shadia Rifai Habbal \\ Institute of Mathematical and Physical Sciences, University of Wales, \\ Aberystwyth, SY23 3BZ, UK, and Harvard-Smithsonian Center for \\ Astrophysics, 60 Garden St., Cambridge, MA 02138, USA
}

Richard Woo

Jet Propulsion Lab, Caltech, 4800 Oak Grove Dr., Pasadena, CA 91109, USA

\begin{abstract}
Identifying the regions of open magnetic structures in the corona, namely regions where field lines expand outwards into interplanetary space, is equivalent to establishing the origin of the solar wind at the Sun. A review of recent studies, based on the comparison of the distribution, as a function of latitude, of density and velocity in the inner corona and in interplanetary space, is presented. It is shown how, at solar minimum, this comparison leads to the unexpected result that the fast solar wind expands indiscriminately from a significant fraction of the solar surface, not limited to polar coronal holes, as has been believed for the past three decades. It is also shown how polarization measurements of coronal forbidden lines, which yield the direction of the coronal magnetic field, lend further support to this result. The implications of these findings are that a significant fraction of the solar magnetic field is primarily open, expanding almost radially into interplanetary space, carrying with it the imprint of the distribution of density in the corona, while the 'closed' structures contribute a small fraction to the overall filling factor of coronal density structures. Furthermore, the solar wind particle flux is found to be correlated with density, implying a higher mass loss rate from the higher density quiet Sun regions, and the likelihood of a solar cycle dependence in the mass loss rate, as the are of polar coronal holes decreases with increased solar activity.
\end{abstract}

\section{Introduction}

One of the consequences of the coronal temperature reaching at least $10^{6} \mathrm{~K}$, as first established from spectral line observations made during total solar eclipses, is the existence of a subsonic-supersonic flow from the Sun into interplanetary space (Parker 1958). Coined the solar wind, the existence of this continuous stream of ionized particles, was soon confirmed in the early 1960's by spacecraft measurements in interplanetary space (Neugebauer \& Snyder 1966). These first conclusive measurements detected streams reaching speeds of up to $700 \mathrm{~km} / \mathrm{s}$, while other streams coasted as slowly as $300 \mathrm{~km} / \mathrm{s}$. In all streams, the density was 
found to be anticorrelated with speed. These basic features of the solar wind were confirmed by all subsequent spacecraft measurements in interplanetary space.

With the advent of space-borne remote sensing observations of the solar corona in the extreme ultraviolet and x-rays in the late 1960's and early 1970's, together with in situ measuring spacecraft, the first breakthrough in the attempts to establish the origin of the fast solar wind at the Sun was achieved. By comparing in-the-ecliptic measurements of the longitudinal variation of solar wind speed made by the Vela and Pioneer VI spacecraft, with the corresponding variation of x-ray intensity at the Sun, Krieger, Timothy \& Roelof (1973) found that wind speeds exceeding $500 \mathrm{~km} / \mathrm{s}$ corresponded to the central meridian passage of the equatorward extension of a polar coronal hole, a region characterized by a minimum in the $\mathrm{x}$-ray intensity. This correspondence was achieved by taking into account the solar wind expansion time and by assuming a radial expansion. Kreiger et al. (1973) thus concluded that coronal holes were the likely source of open magnetic field lines from which the fast solar wind expanded. No velocity or magnetic field measurements in the inner corona were available at the time to validate the existence of an outflow, or of open magnetic field lines, i.e. field lines which extend away from the Sun into interplanetary space.

Theoretical models based on a dipole configuration of the solar magnetic field at solar minimum, provided further supporting evidence for the fastest wind originating from polar coronal holes. In these models, the association of the boundaries of the polar coronal hole with the boundaries of the equatorial streamers, led to the concept of a superradial expansion of polar coronal holes as a prerequisite for fast solar wind.

The launch of Ulysses in 1990 enabled the exploration of the solar wind outside the ecliptic plane up to $\pm 80^{\circ}$ in latitude. During the first full pole to pole pass coinciding with a period of minimum solar activity, fast solar wind was found to fill a significant fraction of the heliosphere, from the poles to approximately $\pm 30^{\circ}$ about the equator (Gosling et al, 1995). This predominance of fast wind was attributed to the earlier model results of the superradial expansion of the boundaries of polar coronal holes.

In what follows, a review of recent studies based on comparisons of the distribution of density and velocity in the inner corona with their interplanetary counterparts from Ulysses measurements, complemented by inferences of the direction of the coronal magnetic field, is presented. Rather than being limited to polar coronal holes and to the superradial expansion of their boundaries, it is shown that the fast wind originates from a significant fraction of the solar surface, encompassing polar coronal holes, equatorial coronal holes and what is commonly known as the quiet Sun. These quantitative comparisons further indicate that, unlike extreme ultraviolet or x-ray observations of the corona, which are biased by the hotter and denser coronal structures (Figure 1a), albeit with a very small filling factor, white light eclipse images of the corona (Figure 1b) yield the most accurate description of the indiscriminate expansion of the solar atmosphere into interplanetary space from the whole solar surface. 

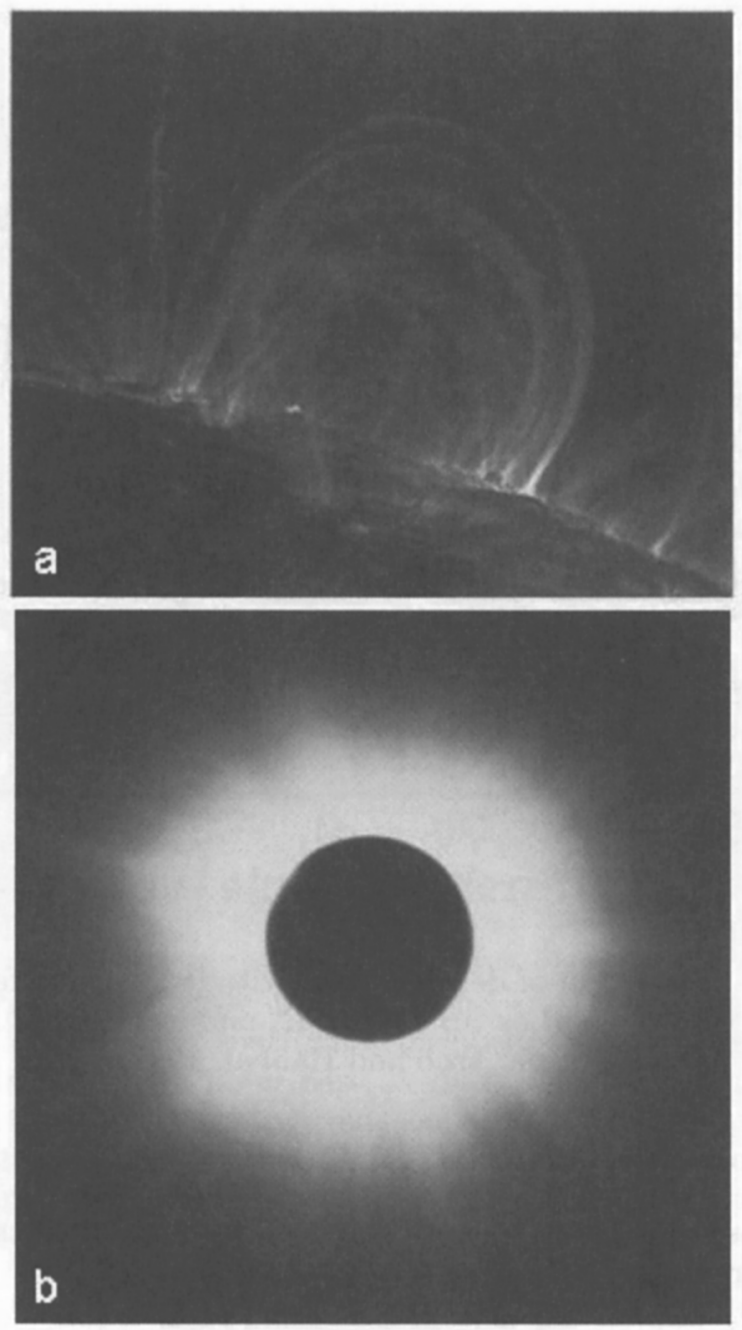

Figure 1. (a) Extreme ultraviolet image of coronal loops taken on 6 November 1999 by the normal incidence telescope on TRACE, in the $17.1 \mathrm{~nm}$ bandpass. (b) White light image of the corona taken during the total solar eclipse of 21 June 2001 


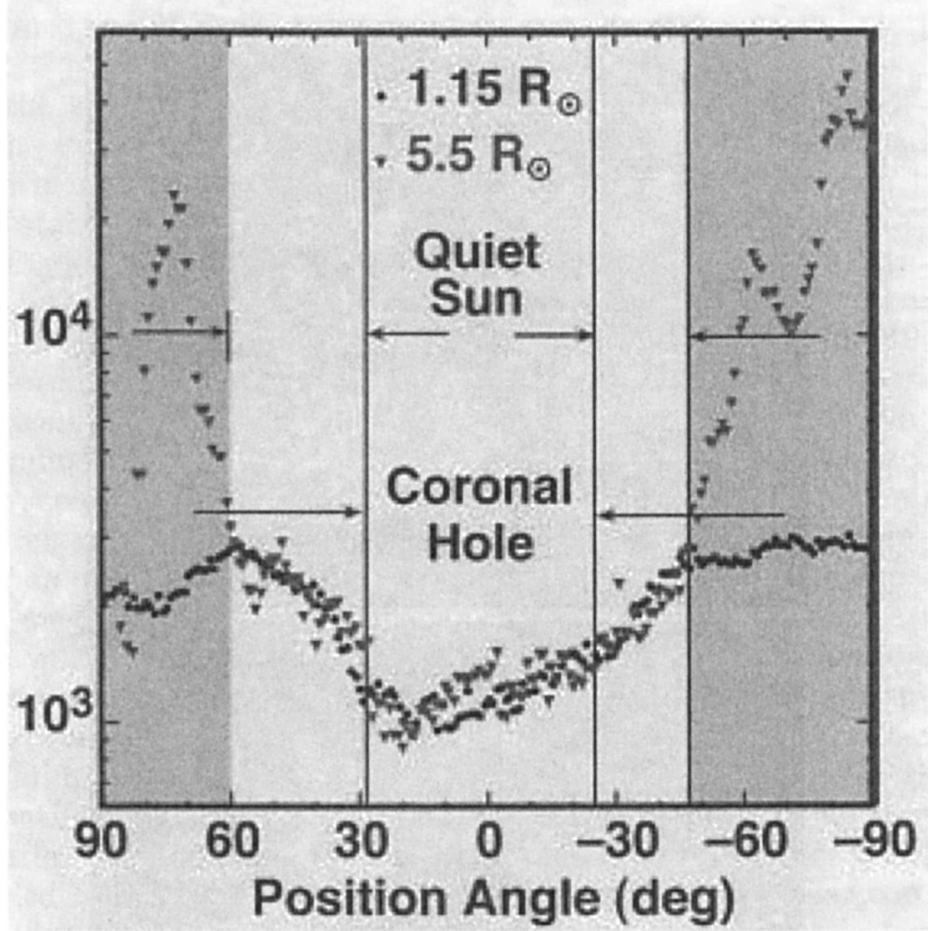

Figure 2. Variation of density with latitude at two different heights in the corona, Mauna Loa $\mathrm{pB}$ at $1.15 R_{s}$ and from LASCO/SOHO C3 at $5.5 R_{s}$. (Adapted from Woo and Habbal, 1999a)

\section{Comparison of Density and Velocity Measurements in the Corona and Interplanetary Space}

Electron density can be readily inferred from measurements of the polarization of the white light emission in the corona. By comparing the latitudinal profile closest to the Sun at $1.15 R_{s}$, measured in the northern hemisphere, with the profile at $5.5 R_{s}$, Woo and Habbal $(1997,1999 \mathrm{a}, 1999 \mathrm{~b})$ found that the relative change of density as a function of latitude was preserved with heliocentric distance (see Figure 2). The preserved profile extended beyond the latitude of the boundaries of the polar coronal hole at the Sun, as identified by disk observations in $\mathrm{He} 1083 \mathrm{~nm}$. The preservation of the latitudinal profile of density with heliocentric distance was interpreted as evidence for a radial expansion of the coronal plasma from both the lowest density polar coronal hole region and the slightly higher density neighboring quiet Sun regions.

Velocity measurements in the inner corona provided further support for this interpretation. Using the concept of Doppler dimming of spectral lines formed by resonance scattering of the disk emission by ions flowing in the corona, 
inferences of flow speeds in the corona (Habbal et al., 1997) were made from measurements of the O VI doublet at 103.2 and $103.7 \mathrm{~nm}$ with the Ultraviolet Coronagraph Spectrometer (UVCS) on SoHO (Kohl et al., 1995, 1997). Using the theoretical estimation that a ratio of 2 in the intensity of these two lines corresponds to a model-independent ion outflow velocity of $94 \mathrm{~km} / \mathrm{s}$, Habbal et al. (1997) produced the first profile of constant flow speed in the inner corona. By measuring the heliocentric distance along this profile, and using it as a proxy for the variation of velocity as a function of latitude, a comparison was made with the latitude variation of density measured at $1.15 R_{s}$ at the same time (see Figure 3, lower panel). These measurements showed that velocity was anticorrelated with density, as found in interplanetary space.

The latitude distribution of velocity and density in the solar wind was also compared with the latitude distribution of fast wind speed and density measured by Ulysses from the first polar pass over the southern hemisphere (Habbal and Woo, 2001) (See Figure 3, top panel). Although not made at the same time, this comparison shows that: (1) the distribution of density with latitude in interplanetary space carries the distinct imprint of the latitudinal extent of the polar coronal hole and the surrounding quiet Sun as measured at the Sun. An increase of about a factor of 2 in density at the latitude corresponding to the coronal hole-quiet Sun boundary, is found to be almost identical to the same increase in the density measurements at $1.15 R_{s}$, at that boundary (see Figure 1a in Habbal \& Woo 2001). (2) The latitudinal distribution of velocity shows a remarkable similarity to the latitudinal distribution of velocity in the inner corona, also measured during solar minimum (lower panel).

Another important result to emerge from the interplanetary measurements was the strong correlation between particle flux (i.e., the product of density and speed) as shown in Figure 4. The average latitudinal profile of density measured at $1.15 R_{s}$ over the southern hemisphere during the same time period of the Ulysses first southern polar pass, is given in the top panel. The Ulysses measurements of density, velocity, and consequently particle flux, were then binned in two latitude ranges (indicated by the vertical swaths in the top panel), one corresponding to $\pm\left(10^{\circ}-20^{\circ}\right)$ from the south pole (left panels), and the other to $\pm\left(40^{\circ}-50^{\circ}\right)$ (right panels). It is clear that the fast wind associated with these two latitude intervals has slightly different plasma characteristics. In particular, the particle flux (or mass loss rate) from the quiet Sun (within the $\pm\left(40^{\circ}-50^{\circ}\right)$ bin is higher than that from the coronal hole $\left( \pm\left(10^{\circ}-20^{\circ}\right.\right.$ bin $)$

\section{Inference of the Direction of the Coronal Magnetic Field from Polarimetric Measurements}

At present, the inference of the direction of the coronal magnetic field remains the only tenable approach to establish its expansion from the photosphere outwards into interplanetary space. Polarimetric measurements of coronal forbidden lines, are the only technique currently available to yield the direction of the coronal magnetic field. The strongest coronal lines suitable for such measurements are the Fe XIV at $530.3 \mathrm{~nm}$ in the visible, and the near infrared Fe XIII line at $1074.7 \mathrm{~nm}$. 

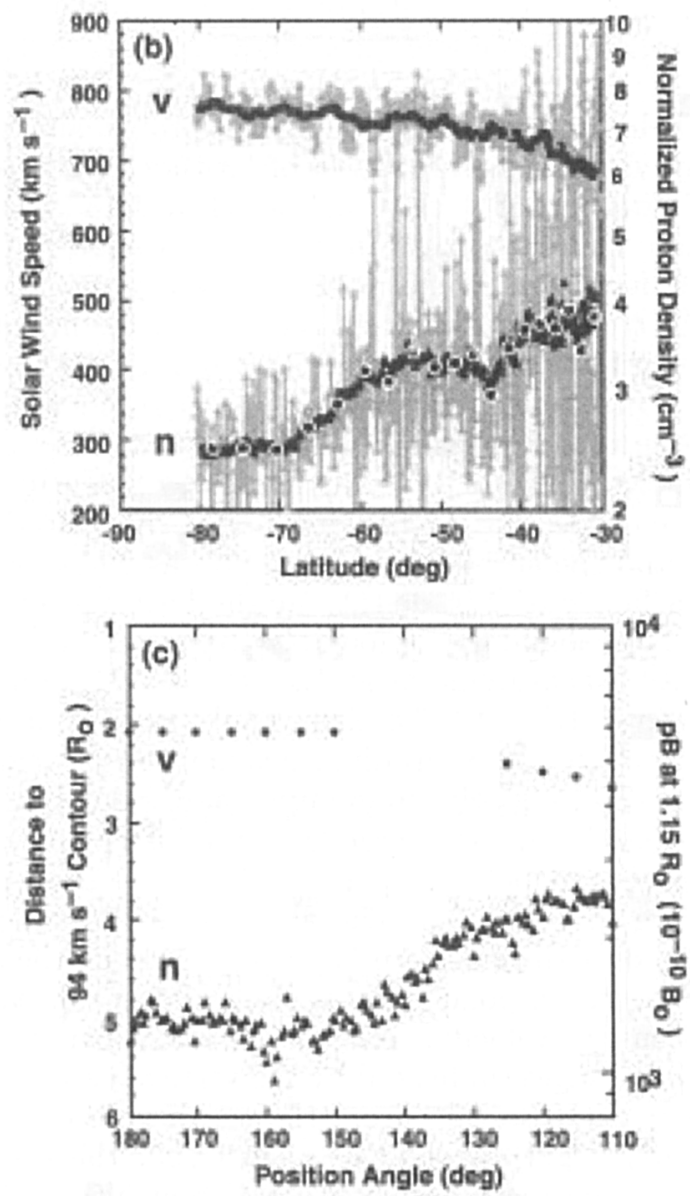

Figure 3. Comparison of velocity and density latitudinal profiles at the Sun (bottom panel) and in interplanetary space (top panel). Bottom panel: $\mathrm{pB}$ at $1.15 \mathrm{R}_{\mathrm{s}}$ and velocity (given by the inverse heliocentric distance of the $94 \mathrm{~km} / \mathrm{s}$ velocity contour level from Habbal et al. (1997)) as a function of position angle, measured counterclockwise from $0^{\circ}$ north, for 17 January 1997. Top panel: daily averages of Ulysses measurements of velocity and normalized density for the fast wind during the slow scan of the south polar region for latitudes $-30^{\circ}$ to $-80.2^{\circ}$ from 3 May 1993 to 15 September 1994. The black circles and triangles represent 27 day sliding window averages. (Adapted from Habbal and Woo, 2001) 

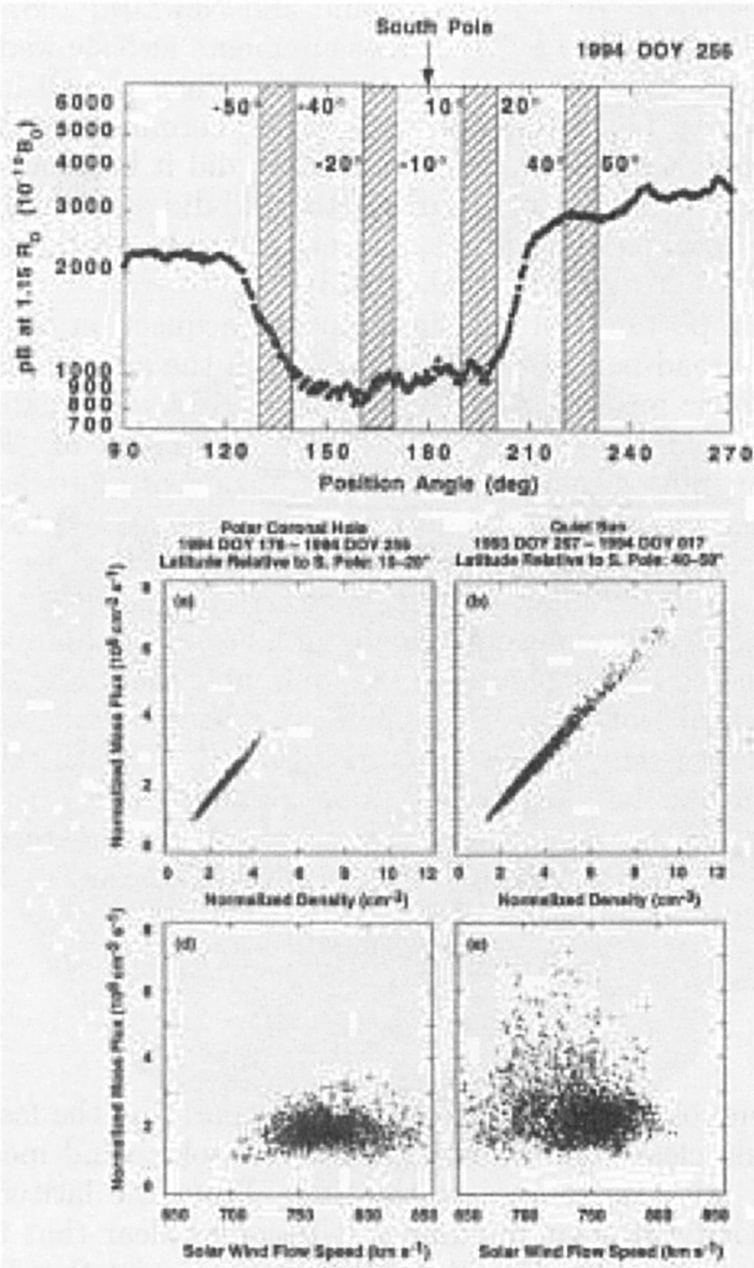

Figure 4. Variation of particle flux with density (middle panels) and velocity (lower panels) for two latitude bins: (left) $\pm\left(10^{\circ}-20^{\circ}\right)$ corresponding to Ulysses measurements from 1994 DOY 170 - 1994 DOY 250 , and (right) $\pm\left(40^{\circ}-50^{\circ}\right)$ for the Ulysses measurements on 1993 DOY 267 - 1994 DOY 017 . The latitudinal density profile at $1.15 R_{s}$ for 1994 DOY 250 is shown in the top panel, with the two latitude bins marked by the hatched areas. (Adapted from Habbal \& Woo 2001) 
The first evidence that the coronal magnetic field expands almost radially from the solar surface appeared in the eclipse Fe XIII polarimetric measurements by Eddy et al. (1973). The only subsequent polarimetric measurements were the KELP Fe XIII observations carried out with the coronagraph at Sacramento Peak Observatory from 1977-1980 (Arnaud and Newkirk, 1987). The maps of polarization angle produced by these measurements also showed a predominant radial direction for the coronal magnetic field. When placed in the context of polarized brightness (i.e. broadband white light) coronagraph observations (see Figure 5), which only became available in 1980, did it become evident that the predominant radial direction of the magnetic field did not match the underlying direction of the brightest structures, namely coronal streamers, (Habbal et al. 2001) as found earlier by Eddy et al. (1973).

Given the importance of the simultaneous acquisition of polarimetric observations with broad-band white light, and with the advance in detector technology, polarimetric measurements in Fe XIII and in white light were obtained during the total solar eclipes of 21 June 2001 (Habbal et al. 2003). Shown in Figure 6, is the resulting map of polarization angle (for more details see Habbal et al. 2003). The resolution in the data was 14" as compared to the average 1.5' in the KELP measurements. The cones labeled A and B, where the polarization direction is consistently radial, correspond to streamers. The regions labeled $\mathrm{C}$ and D correspond to the extension of coronal holes at the Sun, where the polarization was found to be tangential to the limb, and where the Fe XIII emission should have been absent (since its peak formation temperature of $1.8 \times 10^{6} \mathrm{~K}$ exceeds the electron temperature in coronal holes). This unexpected emission was attributed to the fluorescence from silicon nanoparticle dust grains (Habbal et al. 2003). Interestingly, it was the first time that a measurement yielded a marker for the radial extension of coronal hole boundaries, as first detected in radio ranging measurements (Woo \& Habbal 1997).

\section{Conclusion}

Measurements at solar minimum are ideal for separating the fast from the slow wind and making clear connections between the solar wind measured in interplanetary space with its sources at the Sun. From the latitudinal profiles of density and velocity at solar minimum, it becomes clear that the distinct signature of a polar coronal hole and its surrounding quiet Sun is present in the extended corona and in interplanetary space. The imprint seems to be remarkably preserved. While the higher density regions at the Sun produce the slower fast wind, the particle flux is highest from these regions pointing to a higher mass loss rate from a fraction of the solar surface distinct from polar coronal holes, and a potential solar cycle dependence of the mass loss rate as the area of polar coronal holes decreases with increased solar activity.

The preservation of the distribution of density starting from the Sun and outwards into interplanetary space, coupled with a dominant radial direction of the coronal magnetic field, point to an origin for the fast solar wind at the Sun that covers a significant fraction of the solar surface.

These findings resolve some of the puzzling differences between the corona as seen in broad-band white light, and through narrow band spectral line images, 


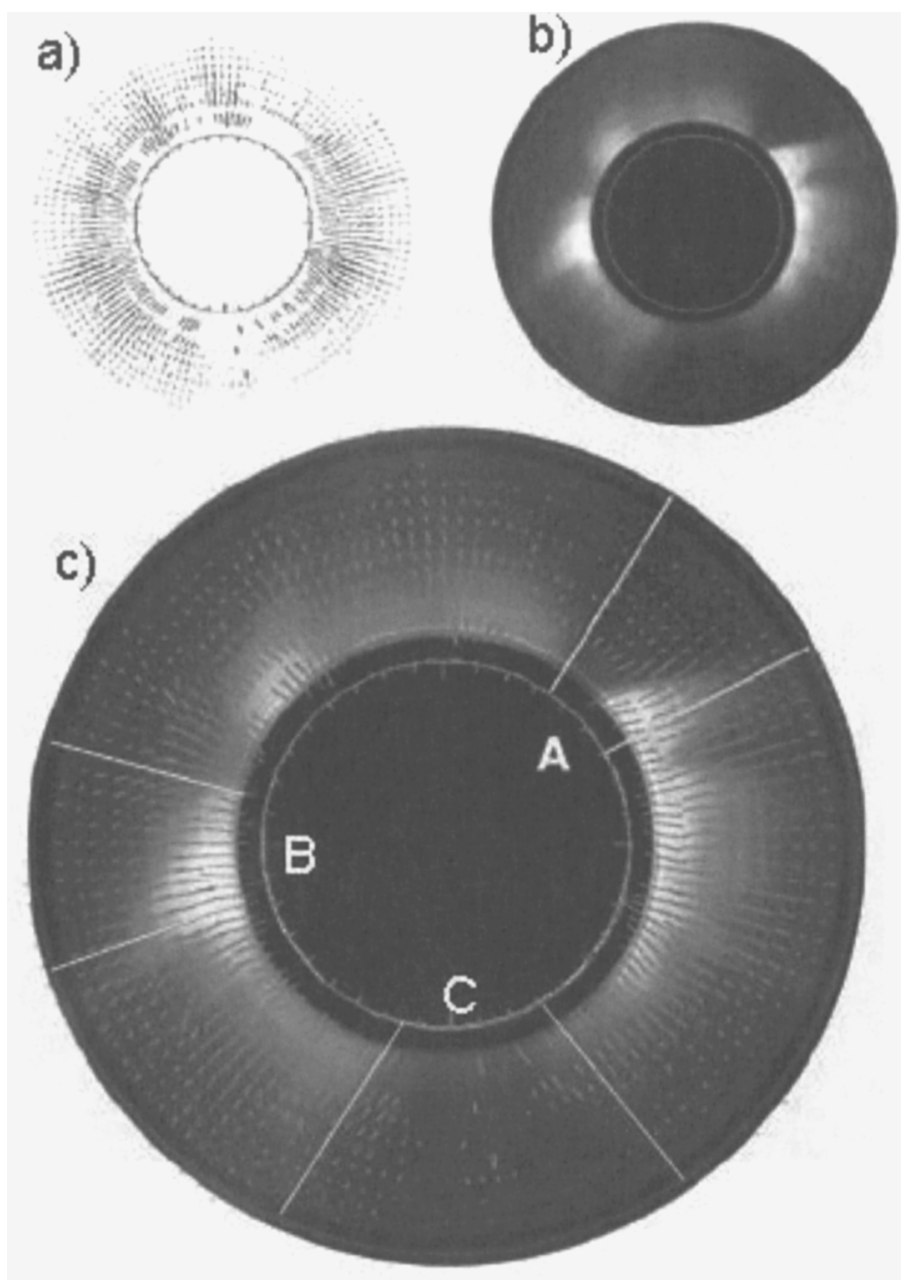

Figure 5. (a) Map of polarization angle of the Fe XIII line emission taken from Sacramento Peak on 1980 February 23. The orientation of the segments gives the direction of the magnetic field, while their lengths are proportional to the polarized intensity $p I$. Tick marks give the position angle in increments of $10^{\circ}$. (b) Corresponding polarized brightness images from the Mauna Loa K-Coronameter. (c) An overlay of the two. The cones labeled A and B isolate a streamer and its vicinity, illustrating how there is no marked difference in polarization direction between the region coinciding with the streamer seen in white light and that directly adjacent to it. The wedge $\mathrm{C}$ isolates the coronal hole region where the emission from Fe XIII is practically absent. (Adapted from Habbal et al. 2001) 


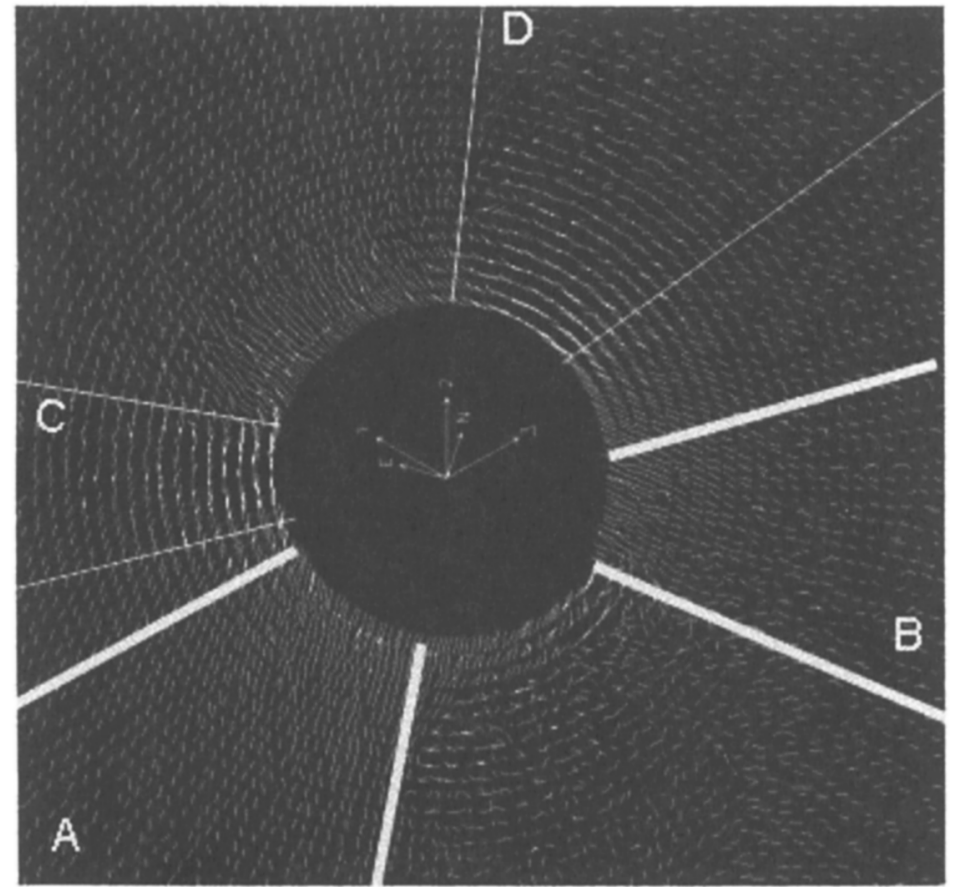

Figure 6. Map of polarization angle from the 2001 June 21 eclipse in the Fe XIII line. The arrows on the moon-occulted disk give the three polarization directions of the polarizer, and the direction of solar north and east. The segments are plotted every $2^{\circ}$ and $0.1 R_{s}$. The length of the polarization vectors is proportional to twice the logarithm of the polarized intensity. 
which are dominated by plasma confined by loop-like structures, albeit with a small filling factor. The coexistence of approximately radially expanding magnetic field lines, with 'closed' magnetic structures, could be a signature of the shallow and deep dynamos found in theoretical investigations (Cattaneo 1999).

Acknowledgments. S R Habbal acknowledges a travel grant from the IAU, and support from NASA grants NAG5-10873 and NAG5-9564 to the Smithsonian Astrophysical Observatory. We thank Dr. M B Arndt for the production of Figure 6.

\section{References}

Arnaud, J. \& Newkirk, G. Jr. 1987, A\&A, 178, 263

Cattaneo, F. 1999, ApJ, 515, L39

Eddy, J. A., Lee, R. H. and Emerson, J. D. 1973, Sol. Phys., 30, 351

Gosling, J. T. et al. 1995, Geophys. Res. Lett., 22, 3329

Habbal, S. R. et al. 1997, ApJ, 489, L106

Habbal, S. R. \& Woo, R. 2001, ApJ, 549, L253

Habbal, S. R., Woo, R., \& Arnaud, J. 2001, ApJ, 558, 858

Habbal, S. R., et al. 2003, ApJ, 592, L87

House, L. L. 1972, Sol. Phys., 23, 103

Judge, P. G. 1998, ApJ, 500, 1009

Kohl, J. L. et al. 1995, Sol. Phys., 162, 313

Kohl, J. L. et al. 1997, Sol. Phys., 175, 613

Krieger, A. S., Timothy, A. F., \& Roelof, E. C. 1973, Sol. Phys., 23,123

Neugebauer, M. \& Snyder, C. W. 1966, J. Geophys. Res., 71, 4469

Parker, E. N. 1958, ApJ, 128, 664

Pneuman, G. \& Kopp, R. 1971, Sol. Phys., 18, 258

Woo, R. \& Habbal, S. R. 1997, Geophys. Res. Lett., 24, 1159

Woo, R. \& Habbal, S. R. 1999a, Geophys. Res. Lett., 26, 1793

Woo, R. \& Habbal, S. R. 1999b, ApJ, 510, L69 


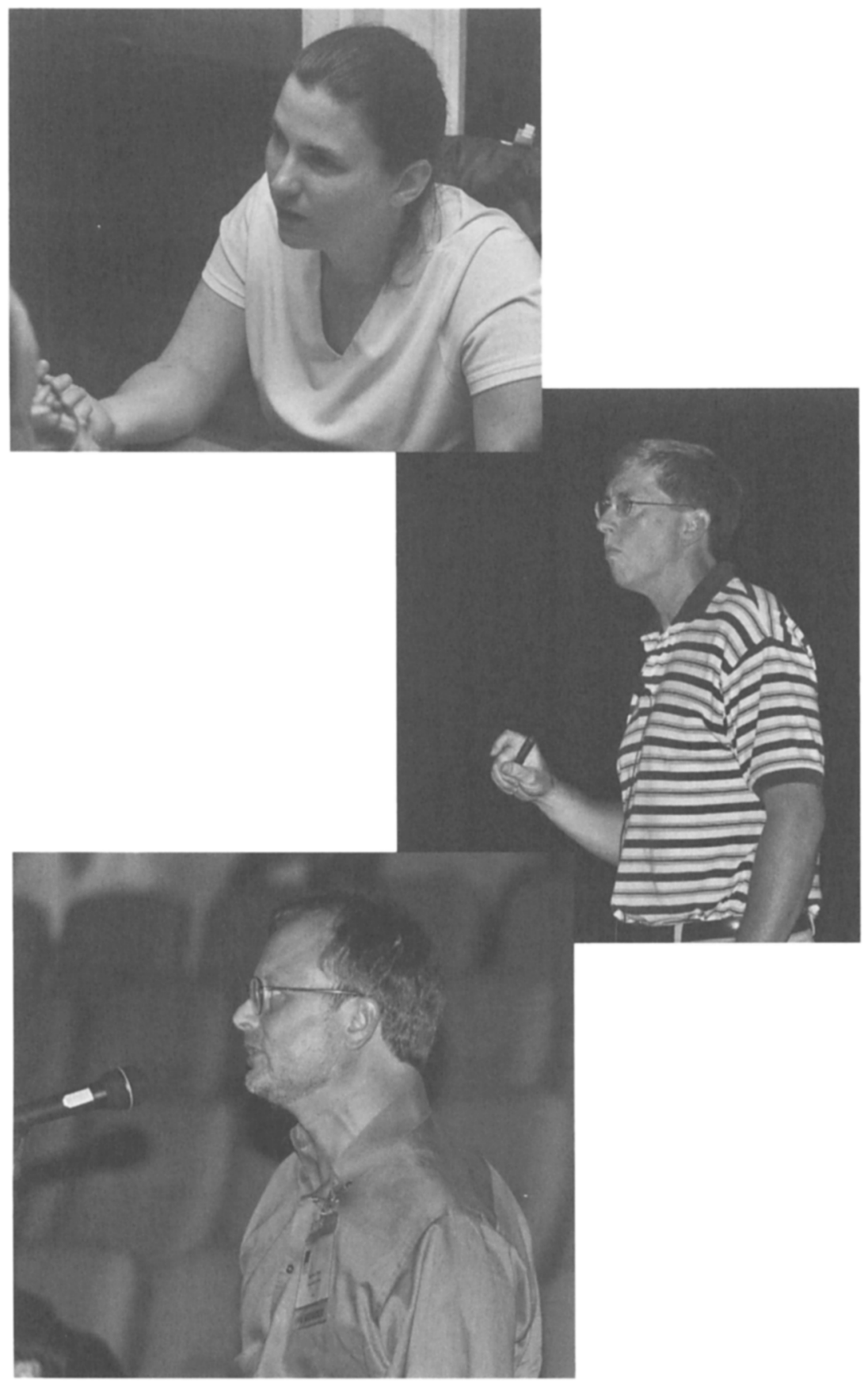

